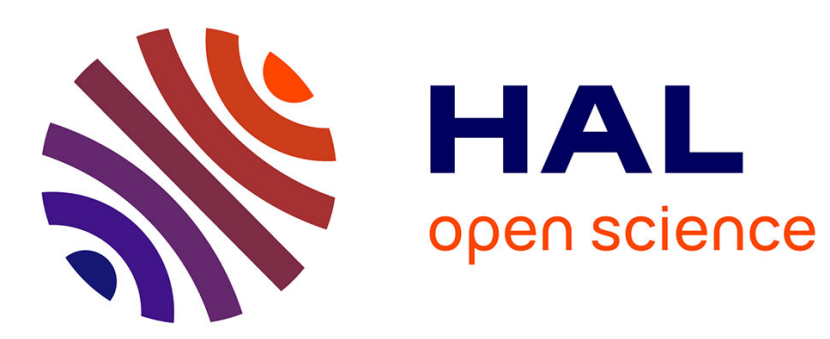

\title{
A parsimonious computational model of visual target position encoding in the superior colliculus
}

\author{
Wahiba Taouali, Laurent Goffart, Frédéric Alexandre, Nicolas P. Rougier
}

\section{To cite this version:}

Wahiba Taouali, Laurent Goffart, Frédéric Alexandre, Nicolas P. Rougier. A parsimonious computational model of visual target position encoding in the superior colliculus. Biological Cybernetics (Modeling), 2015, 109 (4-5), 10.1007/s00422-015-0660-8 . hal-01201785

\section{HAL Id: hal-01201785 \\ https://inria.hal.science/hal-01201785}

Submitted on 18 Sep 2015

HAL is a multi-disciplinary open access archive for the deposit and dissemination of scientific research documents, whether they are published or not. The documents may come from teaching and research institutions in France or abroad, or from public or private research centers.
L'archive ouverte pluridisciplinaire HAL, est destinée au dépôt et à la diffusion de documents scientifiques de niveau recherche, publiés ou non, émanant des établissements d'enseignement et de recherche français ou étrangers, des laboratoires publics ou privés. 


\title{
A parsimonious computational model of visual target position encoding in the superior colliculus
}

\author{
Wahiba Taouali • Laurent Goffart • Frédéric Alexandre • Nicolas P. \\ Rougier
}

Received: date / Accepted: date

\begin{abstract}
The superior colliculus (SC) is a brainstem structure at the crossroad of multiple functional pathways. Several neurophysiological studies suggest that the population of active neurons in the SC encodes the location of a visual target to foveate, pursue or attend to. Although extensive research has been carried out on computational modeling, most of the reported models are often based on complex mechanisms and explain a limited number of experimental results. This suggests that a key aspect may have been overlooked in the design of previous computational models. After a careful study of the literature, we hypothesized that the representation of the whole retinal stimulus (not only its center) might play an important role in the dynamics of SC activity. To test
\end{abstract}

This work was supported by the Institut National de la Recherche en Informatique et Automatique (INRIA), the Centre National de la Recherche Scientifique (CNRS) and the Agence Nationale de la Recherche : grants MAPS (WT, NR, FA and LG) and VISAFIX (LG).

W. Taouali

Institut de Neurobiologie de la Méditerrantée, INSERM, UMR901, Aix-Marseille University, Marseille, France.

Tel.: +33-66-2866453, Fax.: +33-49-1828101

E-mail: wahiba.taouali@inserm.fr

L. Goffart

Institut de Neurosciences de la Timone, UMR 7289, CNRS, Aix-Marseille University, Marseille, France.

F. Alexandre and N.P. Rougier

INRIA Bordeaux Sud-Ouest, Bordeaux, France.

LaBRI, Université de Bordeaux, Bordeaux INP, Centre National de la Recherche Scientifique, UMR 5800, Talence, France.

Institut des Maladies Neurodégénératives, Université de Bordeaux, Centre National de la Recherche Scientifique, UMR 5293, Bordeaux, France. this hypothesis, we designed a model of the SC which is built upon three well accepted principles: the log-polar representation of the visual field onto the SC, the interplay between a center excitation and a surround inhibition and a simple neuronal dynamics, like the one proposed by the dynamic neural field theory. Results show that the retinotopic organization of the collicular activity conveys an implicit computation that deeply impacts the target selection process.

Keywords Computational neuroscience $\cdot$ Neural field · Visual target · Topology · Superior colliculus

\section{Introduction}

The superior colliculus (SC) has been extensively studied over the past decades through an impressive series of neurophysiological experiments that showed its critical role in the gaze orienting response (Anderson et al., 1998; Freedman and Sparks, 1997; Hafed and Krauzlis, 2008; Lee et al., 1988; Munoz and Wurtz, 1995; Robinson, 1972; Schiller and Stryker, 1972; Sparks and Mays, 1980; Sparks et al., 1976). In parallel to these studies, many models have been proposed to explain some aspects of its function (Arai et al., 1994; Badler and Keller, 2002; Droulez and Berthoz, 1991; Gancarz and Grossberg, 1999; Goossens and Van Opstal, 2006; Lefèvre et al., 1998; Marino et al., 2008; Nakahara et al., 2006; Short and Enderle, 2001; Trappenberg et al., 2001). Each model generally emphasized on a specific aspect (or property) of the SC and provided an explanation using a dedicated mechanism. Therefore, a complete picture 
of the contribution of the SC remains very difficult to draw because these models were designed to address specific questions. In this paper, we propose an alternative approach. Instead of adding a new model, we propose a computational model of the SC that gathers the common hypotheses on which most of the previous models were founded. These assumptions are the following: 1-The SC is described as a homogenous map of units with a oncenter/off-surround lateral connectivity. 2-The dynamics of SC activity follows the elementary rules of the neural field theory (Ottes et al., 1986). 3The representation of the visual field locations in the collicular map is non-uniform.

However, in most models, a simplification is made concerning stimulus encoding on the SC map. From the position of the center of the stimulus onto the retina, the log-polar transformation is used to determine the position of the center of the stimulus onto $\mathrm{SC}$ and a shape (and size) is given to the stimulus by setting on that position a circle (Nakahara et al., 2006) or a gaussian (Trappenberg et al., 2001). Particularly, this means that the transformation of the stimulus shape by the log-polar function is not considered in the projection from the retinal map to the SC map. In contrast, an important feature of our model is to consider the projection of the entire stimulus onto the collicular map and not only its center. For the ease of comparison with other models, we kept a simple gaussian shape for the stimulus on the retina. Then, we considered the transformation of this shape by the log polar function as the retinal input onto the SC map. We show, in this paper, that this assumption, combined with the commonly used hypotheses, has powerful emerging effects on the dynamics of the collicular activation.

We confronted the performance or our model against most well-known neurophysiological and behavioral observations and found that these properties could be simply explained in terms of inner population dynamics that scale up to the macroscopic level. In other words, most of the properties of the superior colliculus, particularly those widely studied in target selection paradigms, are observed as emerging from these simple mechanisms. This draws two consequences. First, the proposed model constitutes a solid base for future studies that aim to model the visuo-motor loop. To further assess this proposal, as well as to complement those points that we neglected, sources of the model are made freely available online. Second, this model clearly shows that most experiments are actually measuring the very same low-level properties that differ only in their expression at different scales. Finally, the different simulation tasks reveal that, without taking into account external or high-level inputs, the superior colliculus provides an intrinsic selection process which is dictated by its retinal topology and lateral connectivity.

\section{Model description}

We assume that, in the $\mathrm{SC}$, the locus of activity encodes the position of the stimulus in the visual field. This activity is supposed to feed the oculomotor processes that bring and maintain the stimulus image onto the fovea. Thus, we propose a model composed of two topologically organized layers of neural units. The first layer corresponds to the representation of the visual field in the retina $(R)$ and the second layer to its representation in the deep superior colliculus (SC). As explained before, the model is only built upon assumptions that are common to most SC models:

- The SC is considered as a homogeneous and uniform map of neural units. Its excitatory inputs from the layer $\mathrm{R}$ are transformed by a homogeneous pattern of lateral connectivity characterized by a central excitation whose propagation is counterbalanced by a surrounding inhibition (Arai et al., 1994; Droulez and Berthoz, 1991; Gancarz and Grossberg, 1999; Lefèvre and Galiana, 1992; Nakahara et al., 2006; Optican, 1995; Short and Enderle, 2001; Trappenberg et al., 2001). We assumed a shortrange excitation and a constant inhibition at the whole population level (each unit receives the same amount of inhibition).

- The spatio-temporal evolution of the collicular activation is ruled by a dynamic neural field (DNF) formalism (Amari, 1977) with symmetric connectivity kernels (Marino et al., 2012; Schneider and Erlhagen, 2002; Trappenberg et al., 2001). The activity of a single unit depends upon both the input received from layer $\mathrm{R}$ and the evolution of the population activity in layer $\mathrm{SC}$.

- The projection from the input layer $(\mathrm{R})$ to the output layer (SC) follows a mapping function as described in (Ottes et al., 1986) where a logarithmic function transforms retinotopic coordinates into collicular coordinates, without explicitly modeling its biological substrate 
(Cowey and Rolls, 1974; Daniel and Whitteridge, 1961; Johnston, 1986) . Contrary to other models (Arai and Keller, 2005; Goossens and Van Opstal, 2006; Marino et al., 2012), it is not the center but the whole stimulus which is initially projected onto the SC.

Afferent influences from the cerebral cortex, the basal ganglia, the reticular formation and the cerebellum on the collicular activity are not considered here. Under such conditions, our simulation of $\mathrm{SC}$ activity is limited to those processes that participate in encoding the location of a visual target before a saccade is launched to bring a selected part of its image onto the fovea. Different locations in the visual field are encoded by different loci of activity in the SC (topographical code) (Schiller and Stryker, 1972; Wurtz and Goldberg, 1972) (cf. Fig. 1). Finally, we model one single colliculus and do not consider the interactions between the two colliculi.

\subsection{Visual input}

The space $\mathcal{V}\left[0^{\circ}, 90^{\circ}\right] \times\left[-90^{\circ}, 90^{\circ}\right]$ corresponding to the visual hemifield is mapped onto the space $\mathcal{R}$ $[0,1] \times[-1,1]$ corresponding to a half-retina such that any stimulus presented in $\mathcal{V}$ with polar coordinates $(\rho, \phi)$ (in degrees) is mapped onto $\mathcal{R}$ with Cartesian coordinates $(u, v)$ (in $\mathrm{mm}$ ). We considered round shaped stimuli with Gaussian distribution of luminance such that the activity $U_{r}(u, v)$ at any point $(u, v)$ of $\mathcal{R}$ in response to a stimulus is given by the following equation:

$U_{r}(u, v)=C \exp \left(-\frac{\left|u-u_{s}\right|^{2}+\left|v-v_{s}\right|^{2}}{2 \sigma_{c}^{2}}\right)$

where $\left(u_{s}, v_{s}\right)$ is the center of the stimulus projection onto the $\mathcal{R}$ map, $\sigma_{c}$ the angular size of the stimulus and $C$ is the maximum activation in $\mathcal{R}$ (corresponding to the maximum intensity of the stimulus). Unless stated otherwise, stimuli have an angular diameter (corresponding to the full bandwidth of its Gaussian luminosity at its half maximum (FWHM)) of approximately $1^{\circ}$ in the retina (bandwidth $\left.=1.5 / 90^{\circ}, \sigma_{c}=\frac{\text { bandwidth }}{2 \sqrt{2 \log 2}}\right)$. Gaussian noise $(\mathcal{N}(0,0.01))$ correlated with retinal activation is added to the retinal input to give account of natural noise and for each computation in the SC, Gaussian noise $(\mathcal{N}(0,0.01))$ correlated $^{1}$ with the $\mathrm{SC}$ activation is added to this activation.

\footnotetext{
1 i.e. activation $=$ activation $*(1+\mathcal{N}(0,0.01))$
}

\subsection{Projection of the input onto the SC}

Following the description by Robinson (1972), the projection of $R$ to the SC is approximated using a log-polar transformation, reducing the SC model to a two-dimensional map. A mathematical approximation of this transformation in the monkey was proposed by Ottes et al. (1986) and represents de facto the standard projection used in most computational models (Marino et al., 2008, 2012; Nakahara et al., 2006; Optican, 1995; Trappenberg et al., 2001):

$$
\begin{aligned}
& x=B_{x} \log \left(\frac{\sqrt{\rho^{2}+2 A \rho \cos \phi+A^{2}}}{A}\right) \\
& y=B_{y} \arctan \left(\frac{\rho \sin \phi}{\rho \cos \phi+A}\right)
\end{aligned}
$$

where $(x, y)$ represents the Cartesian coordinates of a neural unit in the $\mathrm{SC},(\rho, \phi)$ the polar coordinates in $\mathcal{R}^{2}$. $B_{x}$ and $B_{y}$ are scaling constants corresponding to the size of the collicular map along the rostrocaudal and mediolateral axes, respectively. The value $A$ is a constant which, together with the ratio $B_{x} / B_{y}$ determines the shape of the mapping. A stimulus at $\rho=90^{\circ}$ and $\phi=-90^{\circ}$ activates a unit at $x=4.8 \mathrm{~mm}$ (corresponding to the length of one colliculus) and $y=-2.76 \mathrm{~mm}$ (corresponding to half its width) (Ottes et al., 1986). As previously explained, the log-polar transformation magnifies the representation of the foveal region (cf. Fig. 1).

\subsection{Dynamic Neural Field}

The SC has been modeled using the DNF theory (Amari, 1977; Taylor, 1999; Wilson and Cowan, 1973) which considers a neuronal volume as a spatial continuum. Although this theory was primarily proposed for simulating activities in the cerebral cortex, the neuronal density within the SC is assumed to be high enough to allow a transposition of this theory to the SC (Marino et al., 2012; Schneider and Erlhagen, 2002; Trappenberg et al., 2001). Thus, the DNF formalism describes the population of active units like a continuous field of activity. The neural units, as elements of the map, are considered as an array of homogeneous processors operating in parallel on incoming

\footnotetext{
2 We do not consider the deformation of the projection from the visual field to the retina since we suppose the transformation used accounts already for that, see Fig1 in Ottes et al. (1986) . Polar coordinates in $\mathcal{R}$ are equivalent to polar coordinates in $\mathcal{V}$,
} 

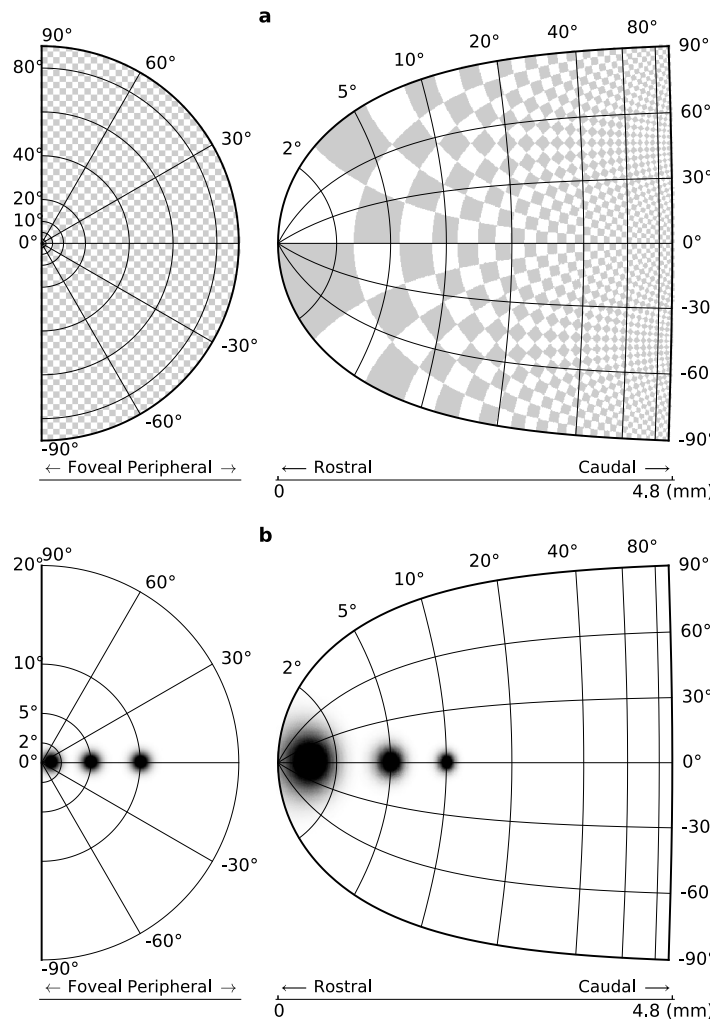

Fig. 1 Projection from layer $\mathbf{R}$ to layer SC. a Retinotopic projection distorts the geometrical properties of the image while keeping the neighborhood relationship. b Conformal mapping from visual space to collicular space makes foveal stimuli to project on a larger area into the superior colliculus compared to more peripheral stimuli

signals. The dynamics of the field activity is governed by an equation of the type:

$$
\begin{aligned}
\tau \frac{\partial \psi}{\partial t}\left(\mathbf{z}_{0}, t\right)=- & \psi\left(\mathbf{z}_{0}, t\right)+S\left(\mathbf{z}_{0}, t\right) \\
& +\int_{\mathrm{SC}} w\left(\left|\mathbf{z}_{0}-\mathbf{z}\right|\right) f(\psi(\mathbf{z}, t)) \mathrm{d} \mathbf{z}
\end{aligned}
$$

where $\psi\left(\mathbf{z}_{0}, t\right)$ represents the activity (i.e. the membrane potential) at position $\mathbf{z}_{0}=(x, y)$ in SC map and time $t, S\left(\mathbf{z}_{0}, t\right)$ represents the synaptic input, $w$ is a weight kernel measuring the strength of connection between two positions, $f$ is the firing rate function of a single neuron and $\tau$ is the time scale factor. The firing rate function is defined by the rectification function $f(\psi)=$ $\min (\max (0, \psi), 1)$ since it is the simplest function that can provide stability for such a field (Hahnloser et al., 2003; Salinas and Abbott, 1996). The density of units in the SC is constant throughout the whole surface and the strength of lateral connections is a function of the distance be- tween units. The lateral kernel of a given unit is thus isotropic (uniform in all directions), contrarily to Nakahara et al. (2006) where the connection strength is asymmetric based on the visual distance between stimuli in the sensory space and not the spatial distance between neurons in the SC map. Even if Nakahara et al. (2006) mention that there is no clear evidence favoring any option, it is also clear that the asymmetric option is a more complex solution to implement and probably more difficult to implement in the underlying population and to justify in terms of biological plausibility. In contrast, the symmetric option, shared with many other models (of the SC and other neuronal structures), underlies a more simple construction of the map. In our model, considering two units at sites $\mathbf{z}_{0}$ and $\mathbf{z}_{1}$ at a distance $\left|\mathbf{z}_{0}-\mathbf{z}_{1}\right|$, the strength of connections is defined by:

$w\left(\left|\mathbf{z}_{0}-\mathbf{z}_{1}\right|\right)=E \exp \left(-\frac{\left|\mathbf{z}_{0}-\mathbf{z}_{1}\right|^{2}}{\sigma_{e}^{2}}\right)-I$

with $E$ being the amplitude and $\sigma_{e}$ the variance of the excitation. For the sake of simplicity, the inhibitory influence is considered as constant throughout the map (see Discussion). The proposed model considers short-range excitatory connections like in several other studies (Arai et al., 1994; Badler and Keller, 2002; Droulez and Berthoz, 1991; Gancarz and Grossberg, 1999; Lefèvre and Galiana, 1992; Nakahara et al., 2006; Optican, 1995; Short and Enderle, 2001; Trappenberg et al., 2001). The effects of local inactivation in the SC were simulated numerically by resetting activity - for the duration of the simulation - in a uniform disc centered on the lesion site $\mathbf{z}_{l}$ with a radius $r_{l}$ corresponding to its extent (i.e. $\forall \mathbf{z}$ in $\mathrm{SC}, \forall \mathrm{t}$, if $\left|\mathbf{z}_{l}-\mathbf{z}\right|<r_{l}$ then $\left.\psi(\mathbf{z}, t)=0\right)$

\subsection{Target encoding}

As reviewed in Gandhi and Katnani (2011), several ways of decoding the SC activity have been proposed. In the present simulations, the kinematics of saccades and their collicular correlates are not considered. In the proposed model, both the static vector averaging and static vector summation can be unified since the SC activity is stereotyped (see Discussion). Unless stated otherwise, the desired eye displacement $\Delta \mathbf{E}$ is decoded using a static vector averaging procedure:

$\Delta \mathbf{E}=\frac{\sum r_{z} \mathbf{R}_{z}}{\sum r_{z}}$ 
where $r_{z}$ is the mean firing rate of a neural unit at a position $z$ and $\mathbf{R}_{z}$ is the preferred vector encoded by that unit taking into account the log-polar transformation. The mean firing rate is defined from equation (2) by:

$$
r_{z}=f(\psi(z, t))(1+\mathcal{N}(0,0.01))
$$

\subsection{Parameters}

The model experiments have been run in Python using the parameters values given in table 1. All sources are available from https://github.com/ taoualiw/Superior-Colliculus.

Table 1 Parameters of the simulations

\begin{tabular}{llll} 
Parameter & Value & Parameter & Value \\
\hline Retina size & $4096 \times 4096$ & Collicular size & $128 \times 128$ \\
$E$ & 1.30 & $I$ & 0.65 \\
$\sigma_{e}$ & 0.1 & $A$ & $3^{\circ}$ \\
$C$ & 1.5 & $B_{x}$ & $1.4 \mathrm{~mm}$ \\
$\sigma_{c}$ & $1.5 / 90$ & $B_{y}$ & $1.8 \mathrm{~mm}$ \\
$\tau$ & $100 \mathrm{~ms}$ & & \\
\hline
\end{tabular}

\section{Results}

\subsection{Population response}

We first investigated the extent of the SC population that is recruited by a stimulus presented at different locations in the visual field. Because of the logarithmic deformation of the visual field onto the SC map, a stimulus that appears in the central visual field initially activates a larger population of units than a more peripheral stimulus (cf. Fig. 1-b). However, due to the pattern of lateral connectivity, the distribution of activity in the map changes over time. After around $250 \mathrm{~ms}$, a stable state is reached and the active population has the same extent regardless of the stimulus location in the visual field, depending only on the ratio between lateral inhibition and excitation in the SC map.

Then, we investigated the profile of the response fields, i.e. the range of target eccentricities that activate a particular location in the SC map. These response fields emerge from the collicular population dynamics combined with the log-polar nature of the input. Indeed, the tuning curves were obtained by recording each site activation level

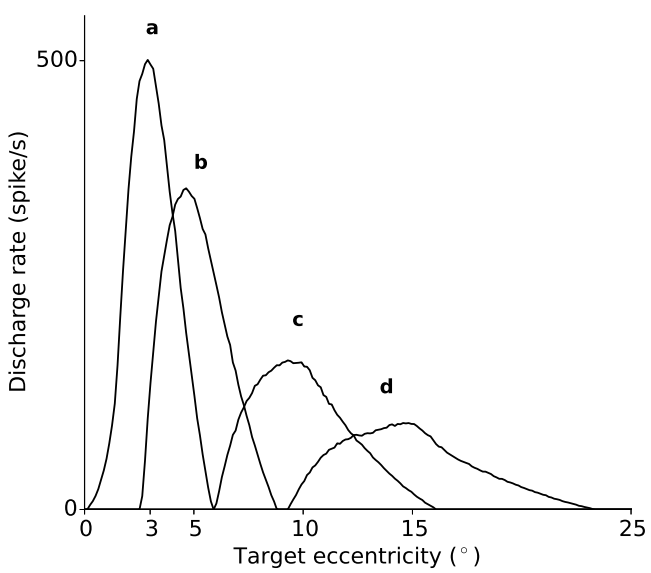

Fig. 2 SC response fields. The tuning curves of SC neurons have been recontructed for sites of maximal activation situated at $\mathbf{A}\left(3^{\circ}, 0^{\circ}\right), \mathbf{B}\left(5^{\circ}, 0^{\circ}\right), \mathbf{C}\left(10^{\circ}, 0^{\circ}\right)$ and $\mathbf{D}\left(15^{\circ}, 0^{\circ}\right)$. The curves were obtained by recording each site activation level (given by the model after stabilization) for each of the 200 stimuli positioned uniformly over $\left[0^{\circ}, 25^{\circ}\right]$. The extent of the response fields decreases with the eccentricity of the stimulus relative to the fovea.

(given by the model after stabilization) for each of the 200 stimuli positioned uniformly over $\left[0^{\circ}, 25^{\circ}\right]$ and not only by applying the projection. The figure 2 shows first that the response field is characterized by a firing rate that is maximal for a specific stimulus eccentricity and that gradually declines for eccentricities going off this preferred eccentricity. Interestingly, the size of the response field is larger and the maximum firing rate lower for units located in the caudal SC than for those located more rostrally.

\subsection{Accuracy and effect of local inactivation}

The model encodes the location of a visual stimulus with an averaging decoding scheme (equation 4). The figure 3 -a shows the actual locations of point stimuli (filled symbols) and the center of mass of their image on the SC (open symbols). In spite of the log-polar transformation and of the expanded response fields, the model exhibits fairly good accuracy (cf. Fig. 3-c,d). For a stimulus presented in the parafoveal visual field, the point image is slightly biased toward the caudal end of the SC, whereas the bias is toward the rostral end when the same stimulus is presented in the peripheral visual field. Small position mismatches are observed when the stimulus point image approaches the rostral and lateral edges of the SC, 

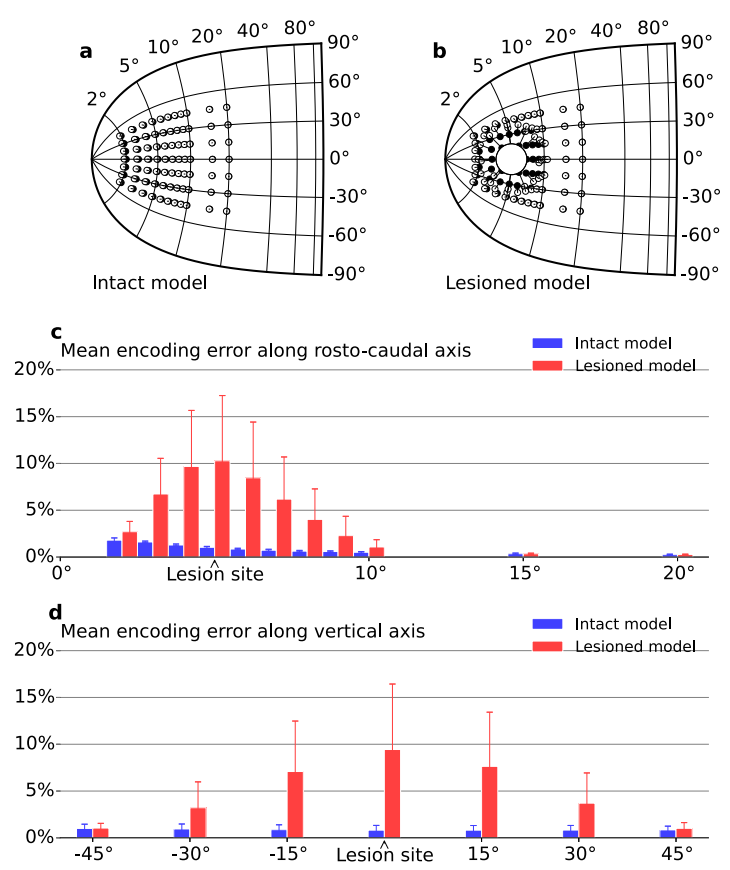

Fig. 3 SC lesions. On left part of the figure, the small open symbols illustrate the center of mass of the stabilized SC activity in response to a stimulus presented at different locations in the visual field (target coordinates are indicated by the small filled symbols). In the intact model (insets $\mathbf{A}, \mathbf{C} \& \mathbf{D}$ ), the relative error in target encoding is less than $2.5 \%$ along both the rostro-caudal axis and the vertical axis with a stronger error in the foveal region $1.8 \%$ that decreases to $0.26 \%$ in caudal positions. However, the mean error is almost constant along the vertical axis (mean $=0.88$, standard deviation $=0.07)$. After a localized inactivation centered at position $\left(5^{\circ}, 0^{\circ}\right)$ (insets $\left.\mathbf{B}, \mathbf{C} \& \mathbf{D}\right)$, there is a dramatic increase in encoding error in the vicinity of the lesioned site

i.e., collicular regions that encode targets located in the foveal field or along the vertical meridian. These are probably artificial distortions due to the fact that we do not take into account the interactions between the two colliculi, as discussed in the concluding part.

We tested the effects of inactivating a small part of the SC (cf section 2.3 for details on the procedure). In the figure 3-b, the inactivation is centered on a site encoding the location $\left(5^{\circ}, 0^{\circ}\right)$ (polar coordinates). A SC activity evoked by a less eccentric stimulus $\left(4^{\circ}, 0^{\circ}\right)$ is shifted toward the rostral $\mathrm{SC}$ whereas an activity evoked by a more eccentric stimulus $\left(6^{\circ}, 0^{\circ}\right)$ is shifted toward the caudal end (cf. Fig. 3-c,d). Because of the inactivation, the symmetry of the population that is normally active is altered; it is re-established by a shift of SC activity away from the lesion. In comparison with
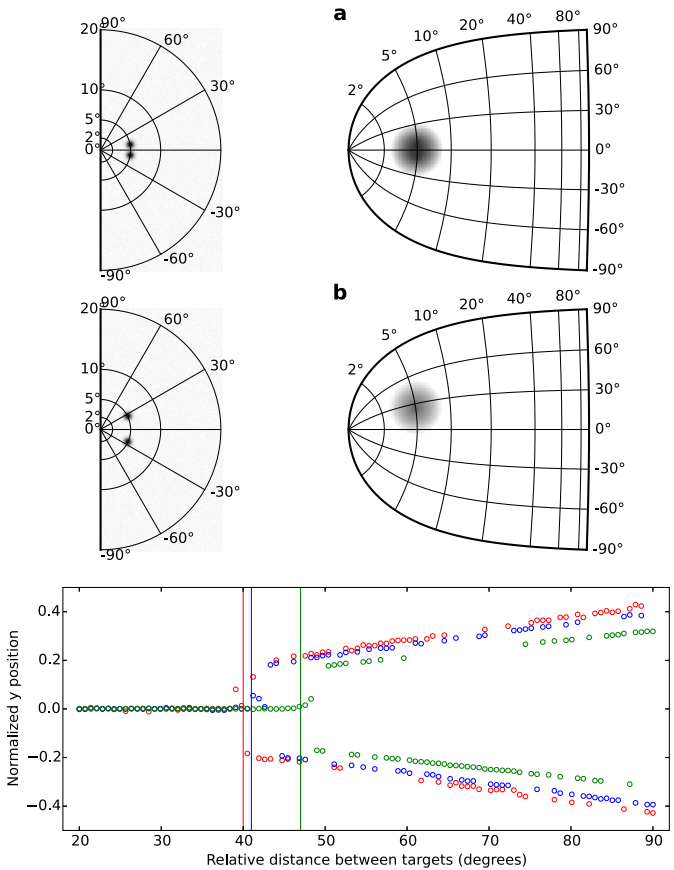

Fig. 4 Double target paradigm. Colored circles correspond to the position of SC activity center as a function of the relative distance between two stimuli presented at same eccentricity and opposite directions. a When the distance is small, the competition between the two stimuli results in an averaging activity in an intermediate position. $\mathbf{b}$ A selection occurs onto one of the two stimuli beyond a relative distance of approximately $40^{\circ}, 41^{\circ}$ and $47^{\circ}$ (vertical lines), corresponding respectively to stimuli at $\rho=15^{\circ}$ in red, $\rho=10^{\circ}$ in blue and $\rho=5^{\circ}$ in green

the time that was required to reach stability before inactivation, the shifted locus of activity stabilizes approximately $50 \mathrm{~ms}$ later, corresponding to an added delay of $20 \%$.

\subsection{Stimuli interaction}

The simultaneous presentation of two visual stimuli activates initially two populations in the SC, which may correspond to an unstable state (Rougier and Vitay, 2006). Starting from an inactive population state, after some time, only one single population remains because the symmetry has been broken by the noise and this leads the system into a stable equilibrium where only one location is active.

The figure 4-a shows that when the angular distance between two stimuli of identical size presented at same eccentricity and opposite directions is small, the population stabilizes at a site which is intermediate between the sites that encode the 
location of each stimulus. For larger angular distances, the population stabilizes at either one of the two sites (cf. Fig. 4-b). Starting from an inactive state (null population activation), this selection is made possible because white noise is added to the activity (see Methods) in order to break the symmetry in the numerical implementation. Interestingly enough, this selection is associated with an increase in the time taken to stabilize the activity at a single site on the collicular map. The figure 4 shows that this limit is higher when stimuli are more peripheral. Indeed, for these stimuli their activation extent is higher and the cartesian distance between them is lower due to the logarithmic projection. The limit value is around $50^{\circ}$ for low eccentricities $\left(\rho=5^{\circ}\right)$ and around $40^{\circ}$ for higher eccentricities $\left(\rho=10^{\circ}, 15^{\circ}\right)$ (cf. Fig. 4).

As shown in figure 6 , the simultaneous presentation of two equivalent targets (same size, same intensity) at different eccentricities leads to a spatial bias towards the more foveal stimulus location due to the logarithmic magnification (cf. Fig.1 ). A selection of the most foveal stimulus occurs beyond a relative distance of approximately $31^{\circ}, 37^{\circ}$ and $43^{\circ}$ (vertical lines), corresponding to the brighter stimulus at $\rho=15^{\circ}$ in red, $\rho=10^{\circ}$ and $\rho=5^{\circ}$, respectively.

This bias could be compensated by increasing the salience of the distal target (size, brightness..). The figure 7 shows the results of presenting two stimuli of same size but at different eccentricities $(\rho$ and $0.75 \times \rho)$ and different intensities (1.0 and 1.5) for three different values of $\rho$ such that the brightness of the more caudal target is $50 \%$ higher than the other. We found that for some specific eccentricity values $\left(\rho=10^{\circ}\right)$ there exists an angular distance threshold $\left(41^{\circ}\right)$ where we got a balanced selection between the two targets (see blue circles in figure 7). However, we found that for the eccentricity values $\left(\rho=5^{\circ}\right)$, there are three stages : an averaging stage, a balanced stage and a biased final stage (see red circles in figure 7). The difference of brightness is not high enough to compensate for the bias of the caudal target. Finally, we found for the eccentricity value $\rho=15^{\circ}$, an averaging stage followed by a biased stage towards the more caudal target. The difference of brightness was so high that it inverted the bias.

\section{Discussion}

The proposed model, based upon a few simple assumptions which are shared by most computa- a

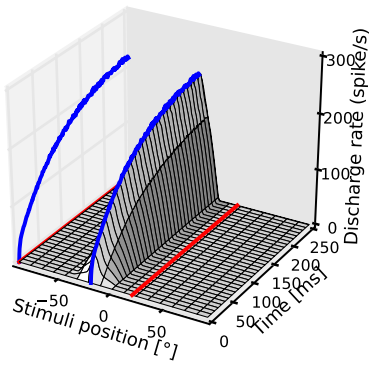

b
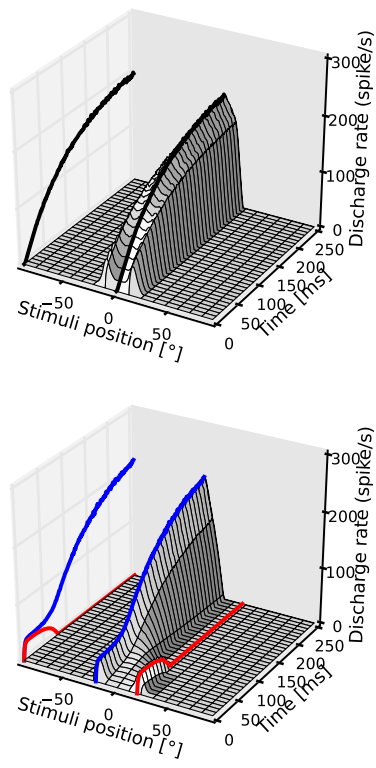

Fig. 5 Double target paradigm I. a Temporal evolution of a slice of $\mathrm{SC}$ for a single stimulus presented at $\left(5^{\circ},+30^{\circ}\right)$. b Temporal evolution of a slice of $\mathrm{SC}$ for two stimuli presented at $\left(5^{\circ},+20^{\circ}\right)$ and $\left(5^{\circ},-20^{\circ}\right)$ showing the merging of SC activity on the central position. c Temporal evolution of a slice of $\mathrm{SC}$ for two stimuli presented at $\left(5^{\circ},+30^{\circ}\right)$ and $\left(5^{\circ},-30^{\circ}\right)$ where the lower stimulus is selected

tional models, can predict a large range of observations made in neurophysiological and behavioral studies. Three core principles characterize this model: the log-polar transformation of the entire retinal input (as opposed to the center of stimuli only), the balance between the excitatory input from the Retina and the lateral interactions (short-term excitation and global constant inhibition) and the dynamics of the population activity.

Our study bears resemblance to the study by Nakahara et al. (2006) showing that an asymmetric kernel led to a spread of activity across the SC (see their figure 3). Indeed, they both share classical hypotheses, particularly representations in to- 

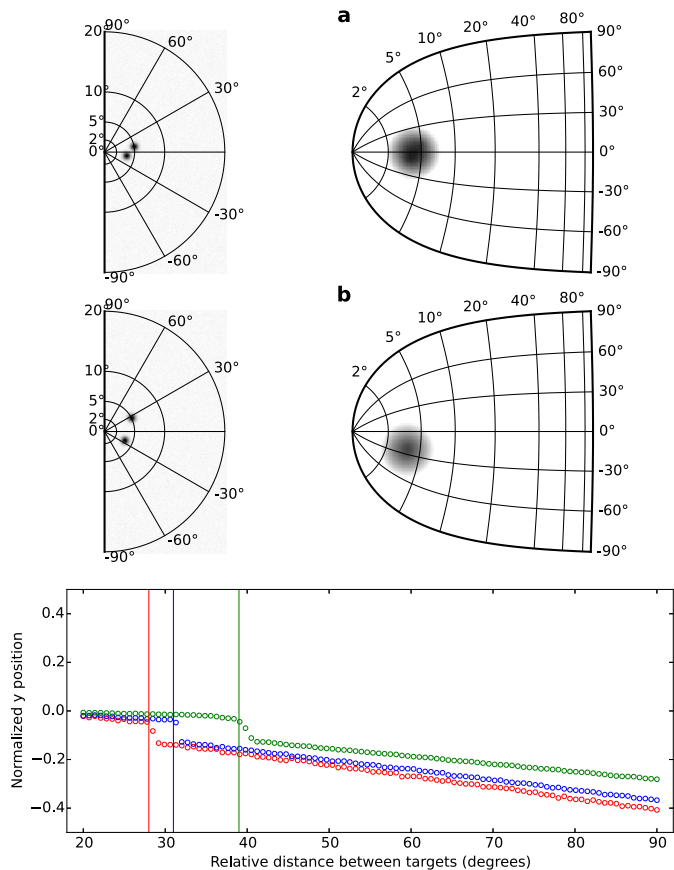

Fig. 6 Double target paradigm II (Eccentricity effect). Two stimuli with same sizes and brightness intensities presented at opposite directions $(-\phi$ and $\phi$, respectively) and shifted eccentricities (0.75 $\rho$ and $\rho$, respectively). Colored circles correspond to the position of $\mathrm{SC}$ activity center as a function of the relative angular distance between two stimuli $(=2 \phi)$ for three different values of $\rho$ ( $15^{\circ}$ in red, $10^{\circ}$ in blue and $5^{\circ}$ in green). a When the distance is small, the competition between the two stimuli results in an averaging activity in an intermediate position. b A selection occurs onto the most foveal stimulus beyond a relative distance of approximately $28^{\circ}, 31^{\circ}$ and $39^{\circ}$ (vertical lines), corresponding to the brighter stimulus at $\rho=15^{\circ}$ in red, $\rho=10^{\circ}$ and $\rho=5^{\circ}$, respectively.

pographical maps (hence similar figures), investigating the model predictions on classical issues (lesioning studies and double target paradigms). Our results are consistent with the absence of spread when using symmetric kernels, however, we were not interested in testing asymmetric kernels in our model for several reasons. First, because it was absent in other studies (Kang and Lee, 2000; Moschovakis et al., 2001) or very moderate (Anderson et al., 1998), it is possible that the spread of activity results from an experimental artefact. Second, to our knowledge, there is no anatomical data supporting asymmetric connection fields in the SC. Finally, Nakahara et al. (2006) recognized that "if the spread is caused by asymmetric intrinsic connections, it may be just an epiphenomenon, with no contribution to movement control".
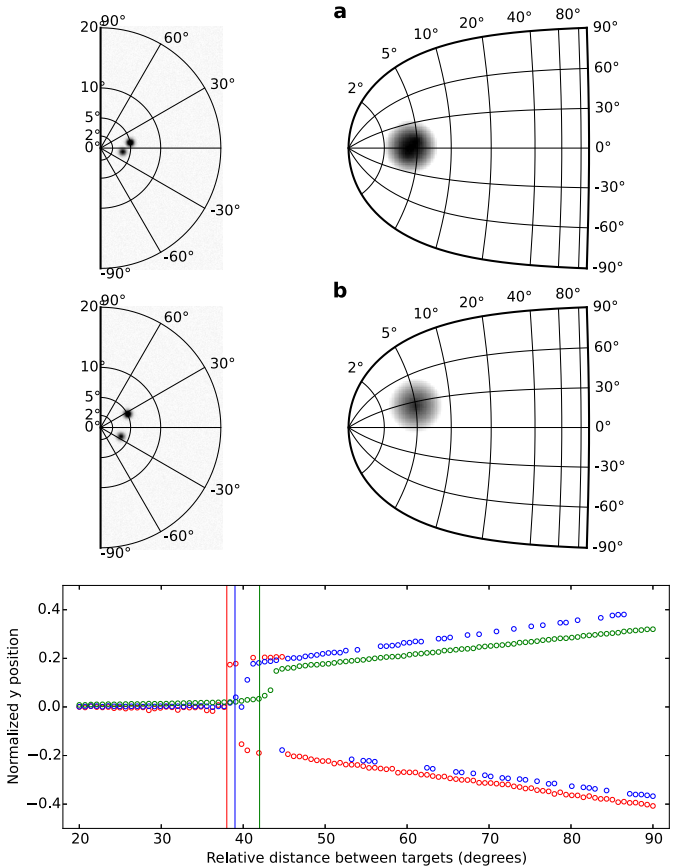

Fig. 7 Double target paradigm III (salience effect: intensity). Two stimuli having different brightness intensities (1.0 and 1.5, respectively) presented at opposite directions ( $-\phi$ and $\phi$, respectively) and shifted eccentricities ( $0.75 \rho$ and $\rho$, respectively). Colored circles correspond to the position of SC activity center as a function of the relative angular distance between two stimuli $(=2 \phi)$ for three different values of $\rho\left(15^{\circ}\right.$ in red, $10^{\circ}$ in blue and $5^{\circ}$ in green). a When the distance is small, the competition between the two stimuli results in an averaging activity in an intermediate position. b A selection occurs onto one of the two stimuli beyond a relative distance of approximately $38^{\circ}, 39^{\circ}$ and $42^{\circ}$ (vertical lines), corresponding to the brighter stimulus at $\rho=15^{\circ}$ in red, $\rho=10^{\circ}$ and $\rho=5^{\circ}$, respectively. The results show that the bias towards the more foveal stimuli locations could be compensated by increasing the salience of the peripheral target (brightness) (i.e., the competition results on the selection of the stimulus with higher eccentricity).

Moreover, a major difference between our model and theirs is the projection of the stimuli. As mentioned in the introduction, Nakahara et al. (2006) used the common assumption of logpolar transformation to determine the position of the stimulus center in the $\mathrm{SC}$, using the projection of the stimulus central coordinates in the retinal map, then, gave empirically a shape to the stimulus by setting on that position a circle. This means that the transformation of the shape of the stimulus by the log-polar function is not directly taken into account, which is a simplification shared with several models. Differently from Nakahara et al. (2006), we propose to project the entire retinal 
stimulus onto the collicular map while keeping the symmetric commonly-used lateral kernels. We show in our experiments that after some cycles of computation, the balance between excitation and inhibition makes the initial distorted activity - corresponding to the projection of the stimulus - become symmetric. Indeed, a major point of our study is to show that this resulting dynamic "transition" is enough to induce powerful emerging properties.

Stabilization onto a unique and stereotyped population In his theoretical study of neural fields, Amari (1977) showed that the neural ensemble evoked by a constant input signal stabilizes onto a unique population of active units with a stereotyped shape, regardless of the position, the size and the strength of the input. Our simulations replicate this phenomenon: the activity evoked by a retinal stimulus stabilizes on a unique and stereotyped population in the SC, regardless of the stimulus size, luminance and position in the visual field. After the initial excitation from the retina, the local excitatory connectivity creates a positive feedback loop, where units activate one another. Two processes contribute to hold this self-sustained excitation on a circumscribed population and to prevent it from growing and spreading further. First, its magnitude is limited by the saturation introduced by the non-linearity in the activation function (implemented in the rectification function). Second, its lateral spread is limited by the constant level of surround inhibition. Regardless of the size and distribution of the initial excitation, the combined effects of the positive feedback loop and the two limiting processes modify the activation profile until the excitation and inhibition balance each other to maintain a stereotyped profile of activation (equilibrium state). The SC has been reported to be the target of endogenous (Lee and Hall, 2006; Meredith and Ramoa, 1998; Munoz and Istvan, 1998; Olivier et al., 1999) as well as exogenous inhibitory influences, e.g., from the substantia nigra pars reticulata (Chevalier et al., 1985; Hikosaka et al., 1983), the central mesencephalic reticular formation (Wang et al., 2010) and the contralateral SC (Olivier et al., 1998). It is worthwhile to mention that, in our model, the question of the origin and distribution of the inhibition is not critical, as the same outcome is observed, regardless of whether it is homogeneously distributed (isotropic) or follows a Gaussian distribution within the SC. The most critical point is that its spatial extent is sufficient large to hold the spread of excitation. Indeed, we assumed a constant inhibition at the whole population level since there is no clear data concerning extended lateral inhibition in the deep superior colliculus. This inhibition could result from efferent influences from other structures such as the basal ganglia. However, this question should be critical for Nakahara et al. (2006) model, where local or global inhibition requires a particular inhibitory connections profile within the SC with no clear consensus for its existence. Finally, the observation that the active population has the same size regardless of the location of the stimulus in the visual field confirms the inferences that were made several decades ago by James McIlwain (McIlwain, 1976). Indeed, this neurobiologist demonstrated that the population of SC neurons activated by a point visual stimulus ought to be constant, regardless of its eccentricity. This translation invariance was later corroborated by recordings of saccade-related neurons in the monkey SC showing that the size of the active area is relatively invariant with saccade size and direction (Anderson et al., 1998; Munoz and Wurtz, 1995).

Changes in accuracy and latency after local inactivation The dynamics described in this study corresponds to the evolution of the activation onto the SC map, from the initial excitation to its stabilization. The duration of the activation increases with the distance between both activation states. This distance depends primarily upon two parameters: the number and the size (spatial extent and magnitude) of the discrete stimuli that feed the SC map. It is also impacted by changes in the activation profile induced by local inactivations. Our results are consistent with the changes in saccade accuracy observed when a small amount of anesthetics (lidocaine) is injected locally in the SC. Indeed, as shown in Lee et al. (1988) study, figure 2, hypometria and hypermetria were observed after injecting lidocaine on one side (or the other) of the location corresponding to the target position. We show similar effects in figure Fig. 3-b; hypometria and hypermetria on the either side of the lesion site. Since our model was not designed to generate saccades, our simulations indicate that this inaccuracy is due to an altered encoding of target location rather than an impaired execution of saccades. It is explained by the dynamics of neural activation that elapses from the initial excitation to its stabilization. The equilibrium state is achieved by a gradual shift of the active population away from the inactivated site. Because the inactivation 
alters the symmetry of activation that normally expands from the center of the initial excitation, the symmetry is re-established away from the lesion, in a more lateral position leading to a change in the topographical encoding of the target location. Because it is aimed at re-establishing equilibrium between the expanding excitation and the surround inhibition, the shift has amplitude and duration that depend upon the overlap between the initial excitation and the lesion. Even though the size of the active population does not depend upon the stimulus size in normal conditions, after $\mathrm{SC}$ inactivation, a stimulus located at the same position in the visual field will be encoded at different sites in the SC, depending upon the size of the stimulus. This model prediction, which is consistent with recent observations (Goffart et al., 2012; Hafed et al., 2008), simply results from the fact that different stimulus sizes lead to different initial excitations in the SC. A larger stimulus initially excites a larger population and the overlap between this initial excitation and the inactivated site determines the trajectory of the SC activity, away from the inactivated neurons, in search for its stabilization. This delayed stabilization could also account for the increase of saccade latencies after SC inactivation (Goffart et al., 2012; Hikosaka and Wurtz, 1985; Sparks et al., 1990).

The response fields of $S C$ neurons The logarithmic projection explains why the response fields of more caudal neurons in the SC have larger width and become more asymmetrical. These properties fit well with the movement field properties described by electrophysiological studies (Munoz and Wurtz, 1995; Sparks et al., 1976). Indeed, the saccaderelated neurons in the $\mathrm{SC}$ burst maximally prior to saccades having a particular direction and amplitude, regardless of the starting position of the eye in the orbit and their resulting tunings are very similar to those resulting from our model (e.g., compare our Fig. 2 with the figure 8 in (Munoz and Wurtz, 1995)). The discharge then declines for other vectors (Schiller and Stryker, 1972; Sparks and Mays, 1980; Sparks et al., 1976; Wurtz and Goldberg, 1972). Interestingly, our model shows how the maximum firing rate declines with the rostro-caudal position of the neuron on the SC map (cf. Fig. 2).

Target selection during image exploration Several studies described the path of saccades generated by subjects exploring various visual images (LévySchoen, 1974; Noton and Stark, 1971; Yarbus,
1967). They showed that the attractive value of an object strongly depends upon its distance relative to the previously fixated object. This attraction is greater for objects close to the fovea (small saccades are more frequent). Such scanpaths do not necessarily rely upon cognitive strategies since our model produces the same performance when its visual input contains multiple identical objects. Because of the log-polar transformation, two visual stimuli of similar size initially activate two populations with sizes that depend on their eccentricity: larger populations are activated when the stimulus is projected near the central region than in more peripheral regions (cf. Fig. 5). The more rostral population wins the competition because of its larger initial size (cf. Fig. 6). The same mechanism can account for the so-called remote distractor effect first reported by (Lévy-Schoen, 1969). When two identical stimuli are simultaneously presented at two different locations, human subjects (Walker et al., 1997) or monkeys (Dorris et al., 2007) favor the stimulus which is closer to the fovea and take a longer time to select this target. Our study shows that the selection simply results from the combined effect of pattern of activity (central excitation surrounded by inhibition) and from the geometrical representation of the visual field within a SC map (magnification of the foveal field).

Temporal dynamics during the competition Using a variety of testing conditions (different target locations, different inter-stimulus intervals), several studies reported increases in saccade latency that were interpreted as resulting from a selection process (Findlay, 1983; Godijn and Theeuwes, 2002; Honda, 2005; Weber and Fischer, 1994). We have discussed above the case where the stimuli were presented at different eccentricities. A more subtle case happens when the two stimuli have the same eccentricity but different elevations. Initially, they activate the same number of units (cf. Fig. 5A,C). However, during a first phase that follows the stimuli presentation, the two corresponding locations in the SC are eligible for selection. Then, the two populations ultimately stabilize onto one single population whose location depends upon the angular distance between the stimuli. If the stimuli are spatially close (cf. Fig. 5A), i.e., if the initial excitations overlap, the two populations merge and stabilize at a site that encodes a location corresponding to the center of mass of the stimuli. Note that this averaging process could also apply to multiple stimuli. Such a merging phenomenon could account for the so-called global effect ob- 
served in saccades made in response to multiple stimuli and distractors (Coren and Hoenig, 1972; Deubel et al., 1984; Findlay, 1982; Ottes et al., 1984; Van der Stigchel et al., 2011). If the two stimuli activate populations in the SC that are separated by a fringe of mutual inhibition (cf. Fig. 5B), the bipolar state could theoretically last forever if the activities were perfectly noiseless and the population is initially inactive. However, the noise added at the level of the visual input introduces fluctuations that break the symmetry and lead to the selection of a single population.

Conclusion The work presented here demonstrates that many properties of the SC activity and the oculomotor behavior can result from the geometry of the retinotopy and the pattern of SC activation (excitation surrounded by inhibition). Our model represents a solid base for investigating further the influence of each afferent input to this brainstem region. It also relies on a small number of free parameters (see Table 1.) which, exception made of the time step of numerical simulations, have a clear biological meaning. This opens many perspectives easy to implement. Firstly, the free parameters can be adapted to fit with neurobiological observations such as the size of receptive fields, the dynamics of SC activation, etc. Secondly, they can also be adapted to fit with behavioral observations or, for a given set of parameters (e.g., mapping functions, levels of inhibition, expanded lesion, visual stimuli of different sizes etc.), to make predictions on the behavioral performance in different species or in patients with different kind of scotoma. These free parameters can be estimated from an optimization process, or be varied systematically, through massive numerical tests. In conclusion, our work shows that behavioral performances that seem complex can result from very simple anatomo-functional principles. It also illustrates the catalytic influence of noise and fluctuations in the irreversible determination of the equilibrium that will encode the location of a target to foveate (Goffart et al., 2012; Hafed et al., 2008; Lee et al., 1988) or to attend to (Zénon and Krauzlis, 2012).

\section{References}

Amari S (1977) Dynamics of pattern formation in lateral-inhibition type neural fields. Biological Cybernetics 27(2):77-87
Anderson RW, Keller EL, Gandhi NJ, Das S (1998) Two-dimensional saccade-related population activity in superior colliculus in monkey. Journal of Neurophysiology 80(2):798-817

Arai K, Keller EL (2005) A model of the saccadegenerating system that accounts for trajectory variations produced by competing visual stimuli. Biological Cybernetics 92(1):21-37

Arai K, Keller EL, Edelman JA (1994) Twodimensional neural networks model of the primate saccadis system. Neural Networks 7(67):1115-1135

Badler JB, Keller EL (2002) Decoding of a motor command vector from distributed activity in superior colliculus. Biological Cybernetics 86(3):179-189

Chevalier G, Vacher S, Deniau JM, Desban M (1985) Disinhibition as a basic process in the expression of striatal functions. i. the striato-nigral influence on tectospinal/tecto-diencephalic neurons. Brain Research 334(2):215-226

Coren S, Hoenig P (1972) Effect of non-target stimuli on the length of voluntary saccades. Perceptual and Motor Skills 34:499-508

Cowey A, Rolls ET (1974) Human cortical magnification factor and its relation to visual acuity. Experimental Brain Research 21(5):447-454

Daniel PM, Whitteridge D (1961) The representation of the visual field on the cerebral cortex in monkeys. The Journal of Physiology 159(2):203221

Deubel H, Wolf W, Hauske G (1984) The evaluation of the oculomotor error signal. In: Gale A, Johnson F (eds) Theoretical and applied aspects of eye movement research, Elsevier, pp 55-62

Dorris MC, Olivier E, Munoz DP (2007) Competitive integration of visual and preparatory signals in the superior colliculus during saccadic programming. Journal of Neuroscience 27:50535062

Droulez J, Berthoz A (1991) A neural network model of sensoritopic maps with predictive short memory properties. Proceedings of the National Academy of Sciences 88(21):9653-9657

Findlay JM (1982) Global visual processing for saccadic eye movements. Vision Research

Findlay JM (1983) Visual information for saccadic eye movements. In: Hein A, Jeannerod M (eds) Spatially oriented behavior, Springer Verlag

Freedman EG, Sparks DL (1997) Activity of cells in the deeper layers of the superior colliculus of the rhesus monkey: evidence for a gaze dis- 
placement command. Journal of Neurophysiology 78(3):1669-1690

Gancarz G, Grossberg S (1999) A neural model of saccadic eye movement control explains task-specific adaptation. Vision Research 39(18):3123-3143

Gandhi NJ, Katnani HA (2011) Motor functions of the superior colliculus. Annual Review of Neuroscience 34:205-231

Godijn R, Theeuwes J (2002) Programming of endogenous and exogenous saccades: Evidence for a competitive integration model. Journal of Experimental Psychology 28(5):1038-1054

Goffart L, Hafed ZM, Krauzlis RJ (2012) Visual fixation as equilibrium: Evidence from superior colliculus inactivation. Journal of Neuroscience $32(31): 10,627-10,636$

Goossens HHLM, Van Opstal AJ (2006) Dynamic ensemble coding of saccades in the monkey superior colliculus. Journal of Neurophysiology 95(4):2326-2341

Hafed ZM, Krauzlis RJ (2008) Goal representations dominate superior colliculus activity during extrafoveal tracking. Journal of Neuroscience 28(38):9426-9439

Hafed ZM, Goffart L, Krauzlis RJ (2008) Superior colliculus inactivation causes stable offsets in eye position during tracking. Journal of Neuroscience 28(32):8124-8137

Hahnloser RHR, Seung HS, Slotine JJ (2003) Permitted and forbidden sets in symmetric threshold-linear networks. Neural Computation 15(3):621-638

Hikosaka O, Wurtz RH (1985) Modification of saccadic eye movements by gaba-related substances. i. effect of muscimol and bicuculline in monkey superior colliculus. Journal of Neurophysiology 53(1):266-291

Hikosaka O, Wurtz RH, et al. (1983) Visual and oculomotor functions of monkey substantia nigra pars reticulata. iv. relation of substantia nigra to superior colliculus. Journal of Neurophysiology 49(5):1285-1301

Honda H (2005) The remote distractor effect of saccade latencies in fixation-offset and overlap conditions. Vision Research 45(21):2773 - 2779

Johnston A (1986) A spatial property of the retinocortical mapping. Spatial Vision 1(4):319-331

Kang I, Lee C (2000) Properties of saccade-related neurons in the cat superior colliculus: patterns of movement fields and discharge timing. Experimental brain research 131(2):149-164
Lee C, Rohrer WH, Sparks DL (1988) Population coding of saccadic eye movements by neurons in the superior colliculus. Nature 332(6162):357360

Lee P, Hall WC (2006) An in vitro study of horizontal connections in the intermediate layer of the superior colliculus. Journal of Neuroscience 26:4763-4768

Lefèvre P, Galiana HL (1992) Dynamic feedback to the superior colliculus in a neural network model of the gaze control system. Neural Networks 5:871-890

Lefèvre P, Quaia C, Optican LM (1998) Distributed model of control of saccades by superior colliculus and cerebellum. Neural Networks 11(7-8):1175-1190

Lévy-Schoen A (1969) Détermination et latence de la réponse oculo-motrice à deux stimulus simultanés ou successifs selon leur excentricité relative. L'Année Psychologique 69(2):373-392

Lévy-Schoen A (1974) Le champ d'activité du regard: données expérimentales. L'Année Psychologique 74:43-65

Marino RA, Rodgers CK, Levy R, Munoz DP (2008) Spatial relationships of visuomotor transformations in the superior colliculus map. Journal of Neurophysiology 100(5):2564-2576

Marino RA, Trappenberg TP, Dorris M, Munoz DP (2012) Spatial interactions in the superior colliculus predict saccade behavior in a neural field model. Journal of Cognitive Neuroscience 24(2):315-336

McIlwain JT (1976) Large receptive fields and spatial transformations in the visual system. International Review of Physiology 10:223-248

Meredith MA, Ramoa AS (1998) Intrinsic circuitry of the superior colliculus: pharmacophysiological identification of horizontally oriented inhibitory interneurons. Journal of Neurophysiology 79(3):1597-1602

Moschovakis A, Gregoriou G, Savaki H (2001) Functional imaging of the primate superior colliculus during saccades to visual targets. Nature Neuroscience 4(10):1026-1031

Munoz DP, Istvan PJ (1998) Lateral inhibitory interactions in the intermediate layers of the monkey superior colliculus. Journal of Neurophysiology 79:1193-1209

Munoz DP, Wurtz RH (1995) Saccade-related activity in monkey superior colliculus. i. characteristics of burst and buildup cells. Journal of Neurophysiology 73:2313-2333 
Nakahara H, Morita K, Wurtz RH, Optican LM (2006) Saccade-related spread of activity across superior colliculus may arise from asymmetry of internal connections. Journal of Neurophysiology 96(2):765-777

Noton D, Stark L (1971) Scanpaths in eye movements during pattern perception. Science 171:308-311

Olivier E, Porter JD, May PJ (1998) Comparison of the distribution and somatodendritic morphology of tectotectal neurons in the cat and monkey. Visual Neuroscience 15(5):903-922

Olivier E, Dorris MC, Munoz DP (1999) Lateral interactions in the superior colliculus, not an extended fixation zone, can account for the remote distractor effect. Behavioral and Brain Sciences 22(4):694-695

Optican LM (1995) A field theory of saccade generation: Temporal-to-spatial transform in the superior colliculus. Vision Research 35(2324):3313-3320

Ottes FP, van Gisbergen JAM, Eggermont JJ (1984) Metrics of saccade responses to visual double stimuli: two different modes. Vision Research 24(10):1169-1179

Ottes FP, van Gisbergen JAM, Eggermont JJ (1986) Visuomotor fields of the superior colliculus: a quantitative model. Vision Research 26(6):857-873

Robinson DA (1972) Eye movements evoked by collicular stimulation in the alert monkey. Vision Research 12(11):1795-1808

Rougier N, Vitay J (2006) Emergence of attention within a neural population. Neural Networks 19(5):573-581

Salinas E, Abbott LF (1996) A model of multiplicative neural responses in parietal cortex. Proceedings of the National Academy of Sciences 93(21):11,956-11,961

Schiller PH, Stryker M (1972) Single-unit recording and stimulation in superior colliculus of the alert rhesus monkey. Journal of Neurophysiology 35(6):915-924

Schneider S, Erlhagen W (2002) A neural field model for saccade planning in the superior colliculus: speed-accuracy tradeoff in the doubletarget paradigm. Neurocomputing 44-46:623628

Short SJ, Enderle JD (2001) A model of the internal control system within the superior colliculus. Biomedical Sciences Instrumentation 37:349354
Sparks DL, Mays LE (1980) Movement fields of saccade-related burst neurons in the monkey superior colliculus. Brain Research 190:39-50

Sparks DL, Holland R, Guthrie BL (1976) Size and distribution of movement fields in the monkey superior colliculus. Brain Research 113(1):21-34

Sparks DL, Lee C, Rohrer WH (1990) Population coding of the direction, amplitude, and velocity of saccadic eye movements by neurons in the superior colliculus. Cold Spring Harbor symposia on quantitative biology 55:805-811

Van der Stigchel S, de Vries JP, Bethlehem R, Theeuwes J (2011) A global effect of capture saccades. Experimental Brain Research 210(1):5765

Taylor JG (1999) Neural bubble dynamics in two dimensions: foundations. Biological Cybernetics 80:393-409

Trappenberg TP, Dorris MC, Munoz DP, Klein RM (2001) A model of saccade initiation based on the competitive integration of exogenous and endogenous signals in the superior colliculus. Journal of Cognitive Neuroscience 13(2):256271

Walker R, Deubel H, Schneider WX, Findlay J (1997) Effect of remote distractors on saccade programming: evidence for an extended fixation zone. Journal of Neurophysiology 78:1108-1119

Wang N, Warren S, May PJ (2010) The macaque midbrain reticular formation sends side-specific feedback to the superior colliculus. Experimental Brain Research 201(4):701-717

Weber H, Fischer B (1994) Differential effects of non-target stimuli on the occurrence of express saccades in man. Vision Research 34(14)

Wilson HR, Cowan JD (1973) A mathematical theory of the functional dynamics of cortical and thalamic nervous tissue. Biological Cybernetics 13(2):55-80

Wurtz RH, Goldberg ME (1972) Activity of superior colliculus in behaving monkey. iii. cells discharging before eye movements. Journal of Neurophysiology 35(4):575-586

Yarbus AL (1967) Eye movements and vision

Zénon A, Krauzlis RJ (2012) Attention deficits without cortical neuronal deficits. Nature 489(7416):434-437 